\title{
Uber eine germanische Lehnwortsippe im Finnischen und Lappischen
}

Im kürzlich erschienenen Teil I seiner »Lautgeschichte der Kainuu-Dialekte» (Kainuun murteiden äännehistoria, 1972, S. 256-257) bringt Alpo Räisänen zahlreiche Belege aus erster Hand für einen Terminus technicus, der in Zusammenhang mit einem in Kainuu wichtigen Gewerbe, dem Teerbrennen, gehört. Es handelt sich um die Benennung für ein einfaches aber praktisches Transportgerät von Teerfässern, dasselbe Wort, das in Lönnrots Wörterbuch in der normalisierten Form palkut: palkkuun auftritt und dem Lönnrot die Bedeutung gibt 'förening af två 1. tre tjärtunnor som sjelfva rulla i st. f. hjul vid transport på bar mark'. Räisänen präzisiert die Definition, indem er dieses Transportmittel skizziert als eine 'Fahrvorrichtung von der Art einer Ackerwalze, wo ein oder auch zwei Teerfässer hinter dem Pferd einherrollen'; in dieser Verwendung ist das Wort häufig ein Pluraletantum, wie u.a. aus den Sammlungen der Wörterbuchstiftung hervorgeht. Räisänen weist gleichzeitig darauf hin, dass das Wort auch eine andere, mit der vorangehenden eng zusammenhängende Bedeutung hat, die primär wirkt: 'zwischen den Gargeln des Fasses anzubringendes gewölbtes Brett, Querleiste, woran die Deichseln der in Frage stehenden Fahrvorrichtung befestigt werden'. Anhand der Sammlungen der Wörterbuchstiftung wird die Art der Befestigung klar: dieses Querholz hatte ein Zweigende als Zapfen, der in das in der Deichsel angebrachte Loch passte und sich drehte, wenn das Pferd die Vorrichtung zog. Die Herkunft des Wortes lässt Räisänen offen; er verweist auf Hakulinen, der $\left(\mathrm{SKRK}^{3}\right.$ S. 269) es unter den autochthonen Wörtern des Finnischen anführt, erwähnt jedoch gleichzeitig, Unterzeichneter habe mündlich den Gedanken geäussert, es könne sich hier um ein Lehnwort handeln. Im folgenden möchte ich diesen 
Gedanken etwas begründen, den ich in einem kleinen Kreis im Zusammenhang mit der Beurteilung der Lizentiatenarbeit von Räisänen i.J. 1968 äusserte.

Wie aus der Darstellung von Räisänen und aus den Sammlungen der Wörterbuchstiftung hervorgeht, ist die Lautvertretung des Wortes in den Dialekten von Kainuu und in deren nächsten Nachbardialekten recht bunt. Sein in den obliquen Kasus auftretender Vokalstamm kann insgesamt sieben Typen vertreten:

1. palakkuve- (in den östlichen Teilen des Dialektgebietes: Paltamo, Ristijärvi, Hyrynsalmi, Suomussalmi, Kuhmo, Sotkamo; ferner in Nordostbottnien im Dialekt von Oulujoki und in Nordkarelien im Dialekt von Nurmes).

2. palakkuvi- (z.B. Gen. Sg. palakkuvin, Nom. Pl. palakkuvit: Paltamo, Ristijärvi).

3. palakkume- (Paltamo, Ristijärvi, Hyrynsalmi, Suomussalmi, Kuhmo).

4. palakkumi- (Nom. Pl. palakkumit: Hyrynsalmi).

5. palakkuo- (Suomussalmi, Kuhmo; NB hierher gehörig Ill. Sg. palakkuìèn palakkù̀ān: Kuhmo).

6. palakk $\bar{u}$ - (im gesamten Dialektgebiet von Kainuu: Paltamo, Hyrynsalmi, Suomussalmi, Kuhmo, Sotkamo, Kajaani, Vuolijoki, Vaala, Puolanka; auch in den westlichen und südwestlichen Nachbardialekten: Kiiminki, Utajärvi, Kestilä, Pyhäntä, Vieremä).

7. palakkūkse- (Kuhmo).

Für den Nom. Sg. liegen nicht sehr viel zuverlässige Nachrichten vor. Es lassen sich drei Typen unterscheiden:

1. palakut (Ristijärvi, ?Kajaani).

2. palaku (Ristijärvi, Kuhmo, Vaala, Oulujoki). Vokalisch lautet das Wort jedoch nur in terminaler Stellung aus; eigentlich handelt es sich um palaku $u^{x}$ mit »Auslautverdoppelung» (fi. loppukahdennus, "jäännöslopuke», »aspiraatio»). Dies zeigt die Vertretung u.a. in den Komposita (z.B. Ristijärvi palaku'?aesa, Vaala palakullauvat, palakukkoukut) und indirekt auch die Flexion (z.B. Oulujoki palaku: Gen. Sg. palakkuven: Partit. Sg. palakutta). 
3. palakkū (Suomussalmi, Puolanka, Vaala, Vieremä).

Zwecks Klärung der Herkunft des Wortes muss untersucht werden, welcher Stammtypus vom Standpunkt der Kainuu-Dialekte als ursprünglich anzusehen ist. Zumindest von den Varianten des Vokalstamms der obliquen Fälle kann ein Teil leicht als sekundär erkannt werden. Somit ist der seltene Typus (7) palakk $\bar{k} k s e-$ deutlich auf der Grundlage des Typs (6) palakk $\bar{u}$ - entstanden (als Muster dienten solche bei den Deverbalen auftretenden Parallelitäten wie makuu- 'Liegen' : makuukse- 'id.; Liegeplatz'), und die seltenen Typen (2) palakkuvi- und (4) palakkumi- sind ursprünglich Pluralstämme der Typen (1) palakkuve- und (3) palakkume-, die in dem als Pluraletantum verwendeten Wort neu umgestaltet sind zu einem Singularstamm. Typus (3) palakkume- wiederum scheint sich aus dem Typ (1) palakkuve- so entwickelt zu haben, dass letzterer einfach zur lautlich nahen Ableitungsreihe überging. Ein Übergang gerade in dieser Richtung lässt sich in den finnischen Mundarten auch sonst manchmal feststellen (verbreitet in den Westdialekten kütkü $(v) e$ - 'Fessel [des Viehs]' $\rightarrow$ kütküme- $\sim$ Südostbottnien kütküme-, wenigstens im Dialekt von Hollola in Südosthäme auch kätkü (v)e- 'Wiege' $\rightarrow$ kätküme-, s. Verf., Vir. 1969 S. 138-139), während Belege für einen Übergang in entgegengesetzter Richtung nicht vorliegen dürften. Der nur aus Suomussalmi und Kuhmo verzeichnete Typ (5) palakkuo- ist wiederum als historisch identisch anzusehen mit dem Typ palakkuve-. Beide Typen setzen nämlich die Vokalverbindung $u-e$ an der Grenze zwischen 2. und 3. Silbe voraus; vgl. kätkï̈ $e^{-}>$in Kainuu allgemein kätküve-, in Suomussalmi und Kuhmo daneben auch kättküö- (näher Räisänen, a.a.O. 252). ${ }^{1}$ - So reduziert sich die Vielfalt der Typen letztlich nur auf zwei: $(1,5){ }^{*}$ palakku(e- und (6) palakk $\bar{u}-$, von denen der erstere in den östlichen Teilen des Dialektgebietes von Kainuu begegnet, der letztere als dessen Parallelform in demselben

${ }^{1}$ Auch der Ill. Sg. palakkù̀ān erklärt sich auf der Grundlage dieses Typs (Angleichung an eine in diesen Gegenden begegnende sekundäre Doppelheit wie porstuva 'Diele': Gen. Sg. porstuvan : Ill. Sg. porstù̀ān = porstu $(t)$; porstuon : porstù̀èn, worüber s. Räisänen, a.a.O. 234). 
Gebiet, ausserdem aber auch in den westlichen Teilen des Dialektgebietes und auch in der Nachbarschaft. Offensichtlich ist gerade der weiter verbreitete Typus palakk $\bar{u}$ - von diesen beiden der ältere. Man kann nämlich aus den Kainuu-Mundarten Parallelfälle aufweisen, wo eine Flexion mit $\bar{u}$-Stamm einer mit uve- bzw. uo-Stamm auswich, jedoch keinen einzigen gegenteiligen Fall. Ein solcher Fall, der ein ursprüngliches $\bar{u}$ voraussetzt, ist ponttū 'fester Ponton aus Stämmen, wo der das Floss ziehende Pferdegöpel liegt'; das Wort hat ein auf das schwedische Original (ponton etwa э: pon'tūn) zurückgehendes $\bar{u}$ in Nordostbottnien und verbreitet in den Savo-Mundarten, einschliesslich Kainuu, ein sekundäres uve, uo (ponttuve-, ponttuo-) jedoch nur in Kainuu (Räisänen, a.a.O. 256). Ein zweiter ähnlicher Fall ist vanttū- 'Wollhandschuh', neben dem aus Kainuu (nur im Dialekt von Kuhmo) vanttuo- aufgezeichnet ist (Räisänen, a.a.0. 257). In beiden Fällen erklärt sich uve bzw. uo als analog; der Übergang von einem Flexionstyp zum andern wurde dadurch ermöglicht, dass die konsonantstämmigen Formen der Wörter vom Typus vanttū-, ponttū- (z.B. Nom. Sg. vantu ${ }^{x} \sim$ vantut : Partit. Sg. vantutta) in den Dialekten von Kainuu gleich aussehen wie die entsprechenden Formen der Wörter vom Typ kätküve- kätküö- (kätkü̈ $\sim$ kätküt : kätküttä). Einen entsprechenden Stützpunkt für den analogen Übergang im Falle von palakk $\bar{u}$ - lieferten der Nom. Sg. palaku und Partit. Sg. palakutta.

Und der Nom. Sg.: hat man sich den Typus (1) palaku bzw. (2) palakut als ursprünglich zu denken oder den den obliquen Kasus entsprechenden langvokalischen Typ (3) palakkū? Im Nom. Sg. des eben genannten Stammes ponttū- begegnet dieselbe Doppelheit (Nom. Sg. pont $u^{x}$ bzw. pontut $\sim$ ponttu $)$, und in diesem Fall ist der Nominativ mit langem Vokal (nicht nur in Kainuu, sondern verbreitet auch in anderen Mundarten belegt) schon von der Etymologie des Wortes her ursprünglich. pontu ${ }^{x}$ (: ponttū-) erklärt sich einfach als Anpassung an den Typ vantu ${ }^{x}$ : vanttu- und das daneben in den östlichen Teilen des Dialektgebietes auftretende pontut als noch jüngere Analogieform, veranlasst durch die in denselben Gegenden vorhandene doppelte Vertretung in den urspr. ut, üt-Nomina und 
nach deren Muster auch im Nomen vantu ${ }^{x}$ (ausser nach gemeinkainuuer Art kätk $\ddot{u}^{x}$, vantu ${ }^{x}$ begegnen hier auch kätküt mit einem erhaltenen ursprünglichem -t und vantut mit einem analogem -t). Der Nom. Sg. palakkū liesse sich theoretisch in gleicher Weise als ursprünglich erklären, doch verfügen wir diesmal nicht über solche etymologischen Beweise wie im Falle ponttū; das Vergleichsmaterial, dass die anderen Mundarten liefern, weist eher auf einen urspr. Konsonantenauslaut hin, wie weiter unten klar wird. ${ }^{1}$ Hinsichtlich jenes konsonantischen Elementes, auf das der Nom. Sg. ursprünglich auslautete, lässt sich aufgrund der Kainuu-Dialekte wenigstens eine negative Schlussfolgerung ziehen: $t$ hat es nicht sein können, denn die Rekonstruktion des Vokalstammes (früherer Typus palakk und nicht *palakku(e-) würde damit in Widerspruch stehen; vgl. die Vertretung der urspr. -t-Wörter: kätküt: kätküve- $\sim$ kätküö-, aber nie kätk $\bar{u}$ - im gesamten Dialektgebiet von Kainuu. Offenbar ist das - $t$ denn auch eine jüngere Vertretung als das Morphophonem $-x$ (= Auslautdoppelung) und erklärt sich ebenso wie das - $t$ des Typs pontut. Wir kommen somit zu dem Schluss, dass die relativ ursprüngliche Flexion des Wortes in den Dialekten von Kainuu den Typus Nom. Sg. ${ }^{*}$ pal $(a) k u^{x}$ : Vokalstamm *pal $(a) k k \bar{u}$ - vertrat. Diese Flexion kann ihrerseits nur auf zwei frühere Typen zurückgehen: e n t w e d e r *palkuh : *palkkuhu- (vgl. in den Westdial. kìru ${ }^{x}:$ kìrū- 'Eile', urspr. *kīruh : *kīruhu-) o d e r *palkus: *palkkuhu- (vgl. in Kainuu und einigen anderen Dialekten vantu ${ }^{x}: v a n t t \bar{u}-$, urspr. *vantus: *vanttuhu-). Gerade die beiden letztgenannten Rekonstruktionen erlauben es, einige Wortelemente der Westdialekte mit dem Wort zu verbinden und die Geschichte der Wortsippe etwas ausführlicher zu erläutern.

${ }^{1}$ Aus der Mundart von Sotkamo ist ein solches Verb wie palakata 'Teer transportieren' aufgezeichnet. Formell lässt sich palakkū als von diesem Verb abgeleitetes Nomen actionis resp. acti erklären, in Wirklichkeit aber ist das in Frage stehende Verb sicher eine auf Volksetymologie beruhende retrograde Ableitung, der als Muster solche im Dialekt allgemeinen Ableitungsverhältnisse dienten wie hakata 'hauen': hakkuu 'Hauen', korjata 'verbessern; ernten': korjuu 'Verbessern, Ernte'. Vgl. die Parallelableitung palakkūkse-, die auf einer ähnlichen Formgebung beruht (oben S. 44). 
Schon in den handgeschriebenen lexikalischen Quellen des 18. Jh. wird das Wort palkus genannt, das 'Untersatz, Block unter dem Amboss in der Schmiede' bedeutet: palkus, pajan 'smidjestubb' (so im interfolierten Exemplar des Wörterbuches von Juslenius, das H. G. Porthan gehörte); palkus, -xen idem ac palkku: pajan palkus = 'smidie stubb' (in Gananders Wörterbuch; in derselben Quelle weiter: palkku idem qvod Tukki, ruhmo, Tolppa 'en tjock Trä klobb där man hugger på; caudex'). Dasselbe Wort lebt auch in den heutigen Dialekten noch. In einem begrenzten Gebiet im Süden von Südostbottnien (Jurva, Teuva, Karijoki, Isojoki, Kauhajoki, Kurikka, Jalasjärvi) und am Nordrand von Obersatakunta (Merikarvia, Siikainen, Hongonjoki, Karvia, Parkano, Kihniö) begegnet zunächst ein Substantiv, dessen Nom. Sg. normalerweise gerade pal(a)kus lautet und das 'aufrechter, runder Block, auf dem hölzerne Gegenstände geschnitzt oder Reiser zerkleinert wurden für das Vieh als Streu' bedeutet, in Parkano auch 'Block unter dem Amboss in der Schmiede'. Als Vokalstamm der obliquen Fälle ist meist pal(a)kkuhu- (Jurva, Isojoki, Kauhajoki, Kurikka, Jalasjärvi, Karvia) bzw. pal(a)kkū- (Siikainen, Karvia, Kihniö) verzeichnet; verglichen damit offenbar sekundär ist der Vokalstamm palkukse-, der nur aus Kurikka genannt ist. Der seltene Flexionstypus scheint noch andere Neuentwicklungen verursacht zu haben: aus Kihniö ist auch der Nom. Sg. pal(a)kkū aufgezeichnet, der auf einer Verallgemeinerung des Vokalstammes beruht, und aus Karijoki, Kauhajoki und Hongonjoki wird der eigentümliche Nom. Sg. palakkus gemeldet, dessen Geminata- $k$ ebenso von dem Vokalstamm palakku(h)uherrühren muss (in Hongonjoki stattdessen allerdings heute das analoge palakkukse-).

Von diesem Gebiet nicht sehr weit entfernt liegt ein anderes, das in erster Linie die Nordgruppe der Südwestdialekte umfasst. Dort besitzt das Wort allgemein zwei parallele Bedeutungen: $a$. 'Block zum Schnitzen oder Hacken (von Reisig, Brennholz)' (wie in Südostbottnien und Nordsatakunta), $b$. 'Querholz, das die Streben vom (Doppel)schlitten verbindet'. Leider wird nur in wenigen Belegen sowohl der Nom. Sg. als auch der Vokalstamm der obliquen Fälle genannt. Ich führe diese Angaben in extenso an: 
Mynämäki palkus : Nom. Pl. palkku 'dickes Querholz, das die Streben am Doppelschlitten miteinander verbindet' (Kustaa Vilkuna 1929).

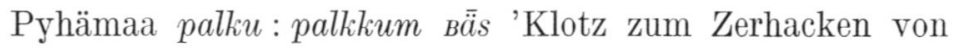
Reisig' (Y. H. Toivonen und Martti Rapola 1928); ferner Nom. Sg. känimsalku 'Holz, das sich um die im Querholz des Vorderschlittens befindliche Nabe dreht' (Martti Rapola).

Pyhäranta palku: Gen. Sg. palkku(n) 'Hackklotz' (Saini Laurikkala 1927).

Laitila palku: Gen. Sg. palkku 'Schnitzklotz', hakopalku 'Klotz zum Zerhacken von Reisig' (J. M. Mikkola 1960).

Mit diesen Aufzeichnungen erfahrener Dialektsammler lassen sich leicht jene lückenhafteren Belege relativieren, aus denen nur entweder der Nom. Sg. oder der Vokalstamm der obliquen Kasus hervorgeht. Im folgenden ein Überblick über diese Angaben (die Buchstaben $a$ und $b$ nach dem Namen der Ortschaft weisen auf die beiden oben genannten Bedeutungen hin): Nom. Sg. palku Askainen $b$, Laitila $a, b$, Kodisjoki $a \sim$ palkus Askainen $b$, Vehmaa $b$, Kalanti $a$; obliquer Stamm palkku- Lappi (Bez. Turku) b (Nom. Pl. palkku[t]), Luvia $b$ (Transl. palkkuks). Die nichtwechselnde starke Stufe der obliquen Fälle zeigt, dass das kurze $u$ der zweiten Silbe auf einen langen Kontraktionsvokal zurückgeht; erwartungsgemäss treffen wir ihn denn auch erhalten an, und zwar in den Gemeinden von Satakunta, die gleich im Nordosten an die nördliche Gruppe der Südwestdialekte grenzen: palkk $\bar{u}$ (aus den obliquen Kasus abstrahierter Nom.Sg.) Köyliö b, palkkū- (obliquer Stamm) Kokemäki $b$, Lavia $b$. Andererseits liegen sowohl aus der nördlichen Gruppe der Südwestdialekte als auch verbreitet aus deren Nachbarmundarten Belege vor, wonach das in Frage stehende Wort einfach Nom. Sg. palkku: Gen. Sg. (soweit erwähnt) palku(n) lautet; vgl. den oben zitierten Beleg aus Gananders Wörterbuch vom gleichlautenden Wort. Die Bedeutung $a$ ist für das Paradigma diesen Typs nur aus Pyhämaa, Laitila und Kalanti aus der Nordgruppe der Südwestdialekte erwähnt; die Bedeutung $b$ (oder die damit eng verbundene 'Querbalken an Wagen ohne Kasten, der die Räder verbindet und als Sitzgelegenheit dient') ist ausser aus der Nordgruppe 
der Südwestdialekte (Merimasku, Paattinen, Mynämäki, Karjala [Bez. Turku], Kalanti, Laitila, Honkilahti, Lappi [Bez. Turku], Rauma, Luvia) auch belegt aus der Gegend von Pori (Pori, Noormarkku, Nakkila), landeinwärts von Turku (Maaria, Lieto, Aura, Karinainen, Pöytyä), Niedersatakunta (Kauvatsa, Kokemäki, Huittinen, Vampula, Loimaa, Mellilä) und stellenweise auch von östlich dieses Gebietes (Tyrvää, Ypäjä, Somero). Aus Ikaalinen in Obersatakunta, unmittelbar von der Grenze des dortigen palkus-Gebietes, liegt ausserdem ein einzelner Beleg vor, wonach palkku einen 'auf Beinen stehenden, waagerechten Holzblock zum Zerhacken von Fleisch' bedeutet. Das Verhältnis dieses Stammtyps zur Flexion vom Typ palku(s) : palkku(u)-, die teilweise im selben Gebiet begegnet, lässt sich schwer entscheiden. Aufseiten der Südwestdialekte, wo die langen Vokale der nichtersten Silben gekürzt sind, könnte es sich nur um eine Verallgemeinerung des Vokalstammes der obliquen Fälle handeln; als vorläufige Erklärung der ausserhalb der Südwestdialekte begegnenden Belege liesse sich wiederum denken, dass man aus einem als schwierig empfundenen seltenen Flexionstyp in einen üblicheren flüchtete, was dadurch verwirklicht werden konnte, dass man sozusagen den "grössten gemeinsamen Faktor» von den Stammvarianten des früheren Paradigmas abspaltete. Die Erscheinung wäre dann eng verwandt mit solchen dialektal begegnenden sekundären Flexionstypen wie kìru 'Eile': Gen. Sg. kìrun (wenigstens in Westuusimaa und in der Gegend von Pori, statt früherem kìru ${ }^{x}:$ kìūn), ori 'Hengst' : Gen. Sg. orin (in der Nordgruppe der Südwestdialekte, in Ostuusimaa und in den Südostdialekten, statt früherem ori ${ }^{x}$ : orhin bzw. o(r)rīn; s. Ruoppila, Kotieläinten nimitykset suomen murteissa I S. 38-44) und neittü 'Jungfrau, Magd': Gen. Sg. neittün (in den Hämedialekten statt früherem neittü ${ }^{x}$ neittü(v)en; s. Verf., Vir. 1969 S. 138).

Wir können nun untersuchen, welche der aufgrund der Kainuu-Mundarten erhaltenen Rekonstruktionen - *palkus: *palkkuhu- oder *palkuh: *palkkuhu- - besser zu den anderen Dialekten passt. Wenn wir von der ersteren Rekonstruktion ausgehen, würde man erwarten, dass das Wort in den verschie- 
denen Dialekten ungefähr ähnlich wie vantus 'Wollhandschuh' vertreten ist, dessen ursprünglicher $s$-Auslaut unbestreitbar scheint. Dieses Wort ist von jeher volkstümlich in den Südwestdialekten und in den daran grenzenden Mundarten von Satakunta, in Teilen von Westuusimaa sowie Süd- und Südosthäme, fast in allen ostbottnischen Dialekten, in den meisten Savo-Dialekten und in den an sie grenzenden Mundarten von Nordwestladoga. In den Südwestdialekten, in Satakunta und Südostbottnien wird es flektiert nach dem Typ vantus (in den Südwestdialekten analog auch vannus) : Vokalstamm der obliquen Fälle vanttuhu- (Südostbottnien, Nordrand von Satakunta $) \sim$ vanttū- (meist in Satakunta) $\sim$ vanttu- (in den Südwestdial.), und eine Flexion entsprechenden Typs ist stellenweise auch in Mittel- und Nordostbottnien bekannt sowie allgemein im äussersten Norden. Wenigstens in den zur Nordgruppe der Südwestdialekte gehörenden Mundarten von Pyhämaa und Pyhäranta begegnet anstelle dieses Typs jedoch das Flexionsverhältnis vantu : vanttu-, was zeigt, dass das Wort in einem bestimmten Stadium überging auf die Ebene der urspr. auf - $h$ auslautenden Wörter (*vantuh: *vanttuhu- ex anal. *kīruh 'Eile' : *kīruhu-, *terveh 'gesund': *tervehe-). Das auf einem entsprechenden Übergang beruhende Verhältnis vant ${ }^{x}$ : vanttu(h)u- ist allgemein in Mittel- und Nordostbottnien und fast ausschliesslich in Kainuu, mit dem Vorbehalt jedoch, dass in den östlichen Teilen des Dialektgebietes von Kainuu auch der analoge Nominativ vantut und der darauf beruhende analoge Stamm obliquer Fälle vanttuo- vorkommt (s. oben S. 45, 46). In den südlich von Kainuu gesprochenen Savo-Mundarten dürfte das Wort verbreitet nur als Pluraletantum bekannt sein; der Vokalstamm lautet überall vanttū-, während der Nom. Sg., der wenigstens in den östlichen und südlichen Teilen der Savo-Dialekte in Gebrauch ist, entweder vantu ${ }^{x}$ oder vantut lautet (sporadisch auch vanttū). Der Typus vantu $u^{x}$ erklärt sich hier aus derselben Analogie wie in den ostbottnischen Dialekten und in Kainuu, während vantut wiederum dessen analoge Weiterentwicklung darstellt: $-t$ pro $-x$ ist hier wie auch in Kainuu ermöglicht worden durch die in den urspr. -ut, -üt-Nomina im selben Gebiet begegnende doppelte Vertretung (Nom. Sg. ent- 
weder kätküt 'Wiege' oder kätkü̈x, während der Vokalstamm der obliquen Kasus kätkiü- lautet). Vielleicht wurde durch das Umgehen des schwierigen und seltenen Flexionstyps seinerseits verursacht, dass das Wort stellenweise in einen ganz anderen Ableitungstyp überging (vanttunen: Nom. Pl. vanttuset in den Dialekten von Nordwest-Ladoga und in den daran angrenzenden Savo-Mundarten) oder flektiert wird wie ein zweisilbiger nichtkontrahierter Vokalstamm (vanttu: Nom. Pl. vantut in Westuusimaa, Südhäme, stellenweise in Südosthäme; weiter nördlich wenigstens in den Mundarten von Jämsä und Keuruu).

Man kann leicht feststellen, dass die Vertretung von palkus bzw. palk $u^{x}$ in den einzelnen Gegenden ziemlich genau der von vantus folgt. Der für die Verhältnisse von Kainuu konstruierte früheste Flexionstyp wie auch die Vertretung des Wortes in Südostbottnien und am Nordrand von Obersatakunta entsprechen genau dem Wort vantus. In den Südwestdialekten begegnet teilweise ein auf $-s$ auslautender Nom. Sg. in der gleichen Art wie in denselben Dialekten allgemein vantus; der einzige Unterschied ist, dass der auf Vokal endende Nom. Sg. palku, der die Vertretung des urspr. Flexionstyps *kīruh: *kīruhun befolgt, daneben in einem etwas ausgedehnteren Gebiet auftritt als der Nom. Sg. entsprechenden Typs vantu, der auf zwei Gemeinden beschränkt ist. Auch der neben diesem Kontraktionsnomen auftretende zweisilbige Vokalstamm palkku entspricht der Bildung vanttu, auch wenn die Gebiete in diesem Fall nicht übereinstimmen. - Vom inneren Stand der finnischen Dialekte gesehen gibt es zwar auch kein direktes Hindernis für die Alternative, dass palkus $\sim$ palku $u^{x}$ zurückginge auf ein auf - $h$ ausgehendes * palkuh. Das palku der Südwestdialekte und das palku $u^{x}$ der Kainuu-Dialekte würden sich dann als erwartungsgemässe Fortsetzungen dieses Typs erklären: begegnen doch $-x$ bzw. in den Südwestdialekten $\varnothing$ anstelle eines urspr. $-h$ in diesen Gebieten auch bei anderen auf - $h$ ausgehenden Nomina, was im Grunde sichtlich eine Angleichung ist an die Vertretung der urspr. - $k$-Nomina. In gleicher Weise erwartungsgemäss wäre aber ebenfalls von einem Auslaut- $h$ ausgehend das in Südostbottnien und am Nordrand von Obersatakunta auf- 


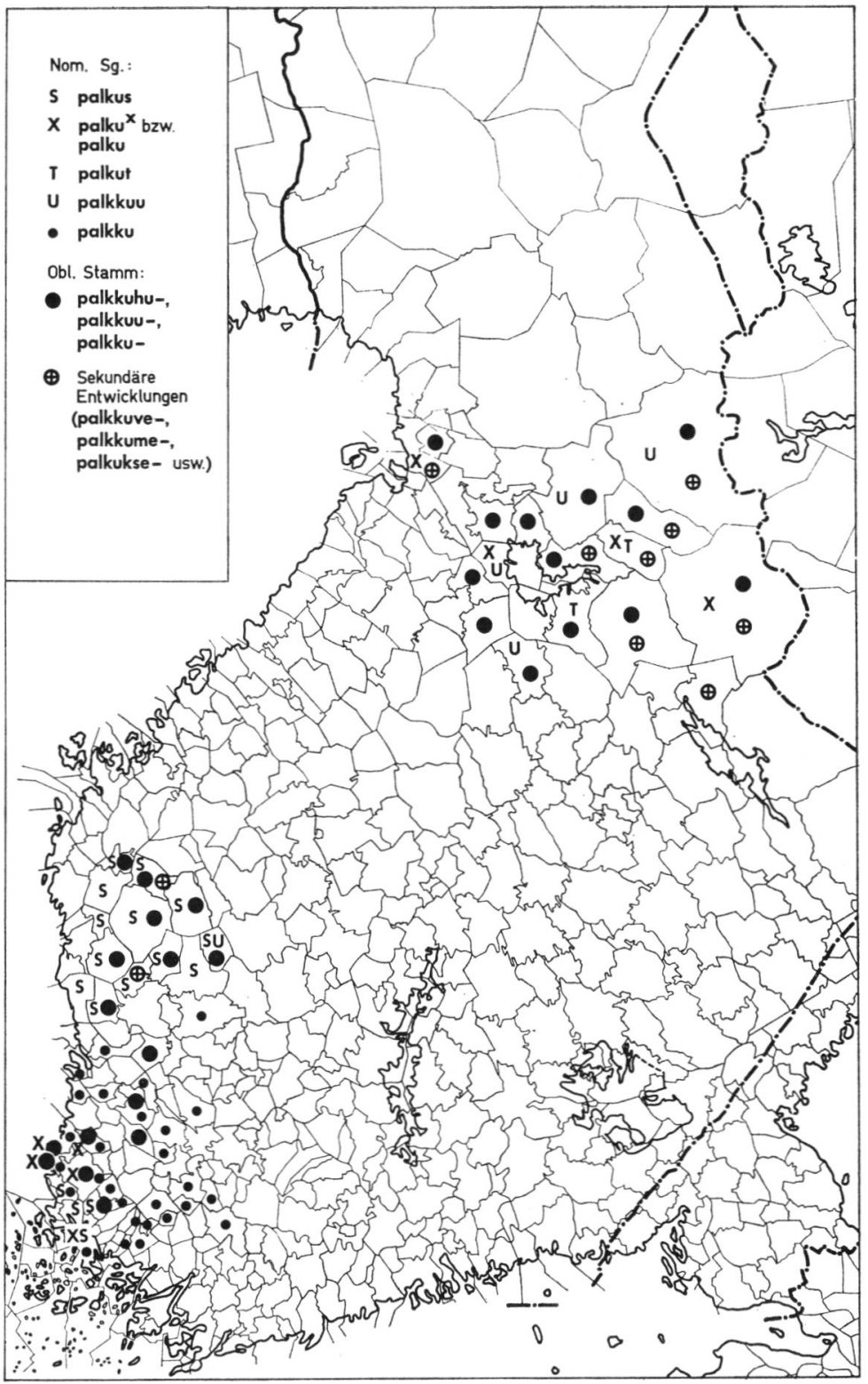

Die palkus-Sippe in den finnischen Mundarten: Varianten des Nom. Sg. und des obliquen Vokalstammes. 
tretende auf $-s$ endende palkus; gerade in diesem Gebiet finden wir ja auch sonst anstelle eines urspr. - $h$ ein $s$, was wiederum eine Angleichung ist an die Vertretung der $s$-Nomina: terves 'gesund', kirus 'Eile' ex anal. kirves 'Axt' : kirvehe-. Das palkus der Südwestdialekte könnte schliesslich auf analoger Angleichung gleichen Typs beruhen, obgleich in diesem Gebiet in anderen $h$-Nomina keine Anzeichen dafür vorliegen dürften.

Erst durch die Etymologie des Wortes neigt sich die Waage entschieden zugunsten eines ursprünglichen $s$-Auslautes. Bekanntlich ist vantus 'Wollhandschuh' ein altes germanisches Lehnwort vom $u$-stämmigen Nom. Sg. *wantuz m., fortgesetzt u.a. in awn. vottr m. (mit erwartungsgemässem $u$-Umlaut in der 1. Silbe), nisl. vöttur m., fär. vøttur m., nnorw. vött u.a., dän. dial. vatt, aschw. vanter, vatter $\mathrm{m}$., nschw. vante (mit sekundärer Angleichung an die schwache Flexion), dial. Jämtland vott. S. z.B. Falk-Torp, Norw.-dän. etym. Wb. und Hellquist, Sv. etym. ordb. sub vante und hinsichtlich der Dialektformen vor allem Gun Widmark, Det nordiska $u$-omljudet I, 1959, S. 234. Eine frühere Gestalt genau desselben Typs lässt sich nun konstruieren für das skandinavische Wort, das u.a. 'Balken' bedeutet und im Neuschwedischen balk lautet (woraus im Fi. die jüngere Entlehnung palkki id. begegnet). Auch dieses Wort geht nämlich in erster Linie auf ein $u$-stämmiges Maskulinum zurück, dessen Nom. Sg. im Urnordischen *balkuz lautete; vgl. awn. (Fritzner) bọlkr, bálkr m. 'Skillevæg, Gjerde; Afdeling, Flokk', nisl. (Bløndal) bálkur m. u.a. 'Skillerum, Skillevæg, som ikke gaar helt op til Loftet; Bænk, ofte af Græstorv, langs Veggen i gamle isl. Folkestuer, ofte anvendt som Sengested; et langt Stykke Træ', fär. (Svabo) bølkur m. 'en stor Hob, Skare, stor Mængde', nnorw. (Norsk ordbok I, 1966) bolk, bokk m. u.a. 'skiljevegg el -planke mellom to rum, t d mellom treskjelåvene og høy- el korn-stål; sterkt gjerde, plankegjerde; stykke, lut, deild ofte om ein (meir el mindre) sjølvstendig lut av ein heilskap', dän. (ODS) balk 'skillevæg, forhøjning', aschw. (Söderwall) balker, bolker m. 'bjälke; balk, afdelning af lagen eller af någon annan skrift', schw. dial. Jämtland bōlk (mit $u$-Umlaut). S. auch Falk-Torp sub bjelke, Hellquist sub balk; hinsichtlich der awn. und aschw. Formen Noreen, 
Aisl. Gr. ${ }^{3}$ S. 66, 241 und Aschw. Gr. S. 311; zur lautlichen Vertretung in verschiedenen nord. Dialekten und deren Erklärung Widmark, a.a.O. 171-174, 304 (nach dieser Quelle sind die awn. Schreibformen bei Fritzner oben normalisiert).

Vom lautlichen Standpunkt kann fi. palkus natürlich in idealer Weise als Entlehnung aus urn. *balkuz erklärt werden. Vom Aspekt des Finnischen her ist gar nicht mal der Nominativ palkus als solcher interessant als vielmehr die Tatsache, dass sich das Wort in seiner Flexion gerade den Kontraktionsnomina angeglichen hat. Im Bereich der fi. auf -us endenden Nomina gibt es für eine solche Flexion nämlich ausser diesem Wort nur noch ein deutliches Beispiel (das oben behandelte Lehnwort vantus: Gen. Sg. vanttuun) und ein weniger deutliches (ruojus: Gen. Sg. ruojuun ${ }^{1}$ in der Bedeutung von 'Schaftschuh, Schaft von Schuhzeug' u. dgl.), während der Typus -us : -ukse- (z.B. sormus 'Ring' : Gen. Sg. sormuksen, otus 'Wild' : otuksen) eine beachtliche Produktivität besitzt. Palkus scheint in diesem Punkt einer Tradition zu folgen, die charakteristisch ist für die ältesten Schichten der auf $-s$ ausgehenden Lehnwörter im Finnischen, und sich ausser in den alten indogerman. Lehnwörtern (z.B. porsas 'Ferkel' : porsaan) und den balt. Lehnwörtern (z.B. hammas 'Zahn' : hampaan, kirves 'Axt' : kirveen) auch widerspiegelt in den german. Lehnwörtern (z. B. varas 'Dieb' : varkaan, laes 'Mahd, Mähbreite, vor dem Schnitter liegender Streifen' : lakeen, kaunis 'schön' : kauniin), ja sogar auch in den lapp. Lehnwörtern (z.B. jokos 'Spur einer Rentierherde oder eines Rentierschlittens im Schnee' : jokkohon $<1 \mathrm{p}$. *čuokkos $=$

${ }^{1}$ Für den urspr. $s$-Auslaut dieses Wortes sprechen vor allem die ostbottnischen Dialekte und ein Teil der Häme-Mundarten; der Vokalstamm wiederum zeigt speziell in Südostbottnien und in den HämeDialekten den für Kontraktionsnomina charakteristischen Typ ruo$j u(h) u$-. Das auf - $t$ ausgehende ruojut begegnet vor allem in den Savodialekten; die Flexion kann auch dann den Typus ruojut : ruojūn aufweisen. Verbreitet finden wir in den verschiedenen Dialekten (sogar in denselben Gegenden, wo ein auf $-s$ bzw. - $t$ endendes ruojus, ruojut fortlebt) auch den zweisilbigen Vokalstamm ruoju oder ruoju $u^{x}$ mit Auslautverdoppelung. Die Etymologie des Wortes ist unklar; vgl. Kettunen, Kalevalaseuran vuosikirja 32 S. 104-109, Virtaranta, Länsiyläsatak. murteiden äh. II S. 705-707, SKES sub ruoju. 
Sk. t'šw $<$ lp. goallos). - Was wiederum die semantische Seite angeht, so lassen sich alle Bedeutungen von fi. palkus mühelos z.B. von jener Bedeutungsgruppe ableiten, die das SAOB für schwed. balk als erstes anführt: '1. stock; föremål som på grund af utseende 1. anv. kan jämföras med en stock: a. — - särsk. om (vågrät) trästock som tjänar till fäste 1. underlag för klenare timmer osv. - - '. Gerade das 'als Unterlage oder Stützholz verwendete Holzstück' ist das gemeinsame Element, das die im Finnischen auftretenden Bedeutungen 'Schnitz- oder Reisigblock, Hackklotz', 'Querholz, das die Streben am Schlitten miteinander verbindet' und 'Querholz, am Boden des Teerfasses befestigt, an dem Deichseln das Fass ziehen' vereint.

Sowohl die ohnehin natürliche urn. Rekonstruktion als auch die Annahme der Entlehnung finden auch im Lappischen Unterstützung. In denselben Zusammenhang gehört nämlich offenbar ein Wort, das nach Friis im Norwegischlappischen in der Lautgestalt balkos: Pl. balkkosak auftritt und 'Stokke, hvorpaa den Stang hviler, hvori Grydekrogen eller andet hænges' bedeutet. Aus dem Norwegischlappischen dürfte das Wort später nicht mehr aufgezeichnet worden sein, doch hat es eine deutliche Entsprechung im Inari-und Skoltlappischen: I (Äimä, JSFOu 25: 5 S. 16, Erkki Itkonen, Ostlp. Vok. S. 160) pâthus : Gen.-Akk. Sg. pailkk'us 'Stange zum Trocknen der Fische', Sk. (T. I. Itk.) Pa pätkp̂s : Gen.-Akk. Sg. pał̀ $k k \hat{n} z$,

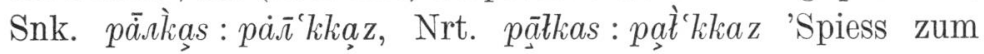
Trocknen von Fischen, (Nrt. auch:) horizontale Stange in der Unterseite der Webevorrichtung der Wolldecken'. Man wird nicht mit Sicherheit entscheiden können, ob das Wort über das Finnische ins Lappische gelangte oder direkt von skandinavischer Seite übernommen ist; im letzteren Falle würde es im Lappischen wie auch im Finnischen sichtlich zu einer sehr alten Lehnschicht gehören, in die Zeit vor der am Ausgang der urn. Epoche eingetretenen Synkope des Vokals der 2. Silbe, während z.B. das aus dem Urn. entlehnte Wort für 'Wollhandschuh', N fac'câ, möglicherweise erst nach jener Synkope übernommen wurde (s. Tryggve Sköld in Scandinavica et Fennougrica, 1954, S. 50-56). 
In genau derselben Bedeutung wie balkos bei Friis erscheint das norwegischlappische Wort balko schon im 18. Jh. bei Knud Leem ('en Stok, tvert over hvilken den Stang hviler, hvorpaa den Trækrog bliver hængt, i hvilken en Søe-Lap haver sin Gryde hængende over Ilden, naar han koger'). Schon Thomsen stellte dies Wort als skandinavisches Lehnwort hin (GSI S. 138); zumindest in diesem Fall braucht das Finnische kaum als Vermittler der Entlehnung angenommen zu werden. Ebenso wenig Bedarf für eine solche Annahme liegt bei den zwei Homonymen des Wortes vor, die von Qvigstad (NL S. 100) ebenfalls als skandinav. Lehnwörter erklärt worden sind: 1. lpS bal'go 'Seilhaspel (von verschiedener Länge)', N bal'ko, I pälkku, Sk. pàl $k k^{A}$ id. (> fi. dial. äusserster Norden palkku id., s. SKES sub palkki); 2. lpTo-N Kvænangen, Kalfj., Lyngen, Balsfj. bal'ko 'abgesonderter Stand für ein Stück Vieh im Stalle', Gullesfj. 'die Wand eines solchen Standes'. Wann wurde nun bal'ko in den verschiedenen Bedeutungen ins Lappische entlehnt? Bindend wird diese Frage schwer beantwortet werden können. Man könnte vermuten, dass die Entlehnung noch nicht lange zurückliegt, wegen der geringen Verbreitung der Wörter und wegen ihrer spezialisierten Bedeutungen, von denen man wenigstens für die beiden letzten (Qvigst. 1, 2) nahe Vergleichspunkte im Neunorwegischen findet. Die Lautgestalt scheint andererseits auch in diesem Fall auf ein altes Lehnwort hinzuweisen. Ein noch nicht synkopierter urn. Akk. Sg. *balku (von *balkuz m.) wäre hervorragend als Ausgangspunkt geeignet; nimmt man jedoch eine nachurn. Entlehnung an, bleibt die Frage offen, weshalb der Stammvokal im Lappischen (o) offenbar genau gerade den urn. Stammtypus des Wortes widerspiegelt. Hans Fromm hat kürzlich (Zeitschrift für dt. Altertum und dt. Literatur 1971 S. 248-249) den Gedanken geäussert, dass zweisprachige Individuen bei der Entlehnung nachurn. synkopierter Formen ins Lappische den etymologisch "richtigen» Stammvokal hauptsächlich aufgrund des Geschlechts der Originale hätten wiederherstellen können, der sich ständig in der Flexion des kongruierenden Adjektivattributs zeigte und teilweise auch im Substantivparadigma selbst: daher z.B. -o normalerweise in den ins Lappische entlehnten 
Feminina, - $a$, - $\hat{a}$ oder $-e$ in den Maskulina und Neutra. Das Auftreten von -o in den lappischen Entsprechungen einer solch kleinen Wortgruppe wie der urspr. $u$-stämmigen Maskulina (vgl. auch z.B. lp. bar'ko 'Baumrinde' awn. borkr) kann durch diesen Mechanismus jedoch nicht ohne weiteres erklärt werden; der "richtige» Auslautvokal müsste wohl entweder auf dem Zufall beruhen oder vielleicht auf einer Abstraktion aus solchen awn. Flexionsformen mit $u$ wie Akk. Pl. *bolku, Dat. Pl. *bolkum (von bolkr m.). Auch die letztere Alternative dürfte überhaupt nur unter der Voraussetzung möglich sein, dass das awn. $u$-umgelautete $o$ aus früherem $a$ noch zur Zeit der Entlehnung ein dem $a$ nahestehender Vokal war (zur Begründung dieser Möglichkeit s. Tryggve Sköld, Die Kriterien der urn. Lehnwörter im Lappischen I S. 187). Qvigstad führte von dem Wort für 'Seilhaspel' auch solche Varianten an, wo sich im Vokal der ersten Silbe der $u$-Umlaut widerspiegelt: lpS Hatfjelldal bolkö, To. Lenvik boal'ka, Ibestad, Ofoten boal'kō. Hierbei könnte es sich um eine - verglichen mit dem obigen Wort - spätere Entlehnung aus awn. bolkr handeln, wenn nicht direkt aus nnorw. bolk.

Auch wenn lp. bal'ko gar nicht auf fi. Einfluss zurückgehen können wird, lässt es uns doch noch einmal nach fi. palkku blicken, dessen Entstehungsgeschichte oben in gewissem Ausmass unklar blieb. Im selben Zusammenhang, wo Thomsen das von Leem genannte lp. Wort balkko behandelte, sah er bereits auch in diesem fi. Wort unter Vorbehalt ein germanisches Lehnwort; ihm war das Wort in der Bedeutung 'caudex sectorius' bekannt, wie das Wörterbuch von Renvall sie anführt. Gleichfalls zögernd hat T. E. Karsten diese Zusammenstellung wiederholt (Finnar och germaner, Folkmålsstudier 10, S. 364); er sah in dem Wort ein »fi. Deminutivum zu palkki od. mögl. ein urn. *balku- in isl. bolkr». Es besteht kaum Anlass, das Wort als Deminutiv von palkki aufzufassen; dagegen kann zumindest für möglich gehalten werden, dass es sich nicht um eine auf fi. Grundlage entstandene „Verstümmelung» auf der Basis etwa eines früheren Verhältnisses ${ }^{*}$ palku $\left({ }^{x}\right):{ }^{*}$ palkk $\bar{u}$ - in oben umrissener Art handelt, sondern um eine direkte Entlehnung von skandinavischer Seite. Im letztgenannten Fall müsste auch im 
Finnischen an einen ähnlichen Ausgangspunkt gedacht werden wie für lp. bal'ko: diesmal entweder urn. Akk. Sg. *balku oder vielleicht aschw. Dat. Pl. *balkum bzw. Akk. Pl. *balku (von denen zwar der letztere einen in der aschw. Flexion der $u$ Stämme seltenen Typus verträte, statt des gewöhnlicheren auf - $i$ endenden Typus Akk. Pl. syni 'Sohn', s. Noreen, Aschw. Gr. S. 312).

Abschliessend verdient die Tatsache Erwähnung, dass die Vielfalt der Typen im Finnischen auch hiermit noch nicht aufhört. Wie u.a. im SKES sub palkki kurz erwähnt wird, begegnet im Fi. auch das $a$-stämmige palkka, das offenbar zur selben Wortsippe gehört wie palkus, palkku und palkki. Dieses Wort ist als solches in den nördlichsten Teilen des finnischen Sprachraums bekannt (die südlichsten Belege des einheitlichen Verbreitungsgebietes stammen aus Simo, Pudasjärvi und Kuusamo) und bedeutet dort eine waagerechte Stange, eine Latte, an der runde Roggenbrote mit einem zu diesem Zweck in der Mitte angebrachten Loch (fi. reikäleipä 'Lochbrot') aufbewahrt oder z.B. Fleisch, Fisch, Wäsche getrocknet werden. Nach mehreren übereinstimmenden Angaben war das Wort ausserdem von jeher auch weiter südlich in Gebrauch, in Nordsavo im Dialekt von Nilsiä (palakka 'Latte zum Trocknen z.B. vonKleidern und Fellen'). Am Südrand des Sprachraums lebt das Wort wiederum im Deminutivum palkkanen: Pl. palkkaset 'die Sparren in der Riege beim Ofen, die kürzer sind als die anderen Latten' (Südhäme) und im Kompositum palkkaparsi id. (Somerniemi in Südwesthäme) sowie in zwei Weiterbildungen: palkkalainen 'kurze Sparre in der Riege' (Westuusimaa) und palkkamies 'kleiner Keil, benutzt z.B. zum Anziehen des Reifens um den Zuber' (Niedersatakunta). Die beiden letztgenannten Wörter sind offenbar scherzhafte Bildungen, veranlasst durch die auf dem Homonym palkka 'Lohn' beruhende Ableitung palkkalainen 'Lohnempfänger; Tagelöhner' und das gleichbedeutende Kompositum palkkamies. Theoretisch könnte auch dieses palkka zurückgehen auf die Fortsetzung vom Wort *balkuz, und zwar zunächst auf die anzunehmenden aschw. Formen *balkar, *balka, die regelmässige Formen des Gen. Sg. bzw. Pl. in der aschw. Flexion eines $u$-stämmigen Maskulinums wären. 
Näher liegt aber vielleicht doch der Gedanke, dass fi. palkka auf die eine oder andere Weise denselben germanischen Stamm *balkan- widerspiegelt, der insbesondere für die westgerman. Sprachen gilt (s. Kluge-Mitzka, Etym. Wb. der dt. Sprache sub Balken), dessen Fortsetzung jedoch auch auf skandinavischer Seite festzustellen ist: aschw. balke $\mathrm{m}$. neben dem oben behandelten balk m. (s. SAOB und Hellquist sub balk, Söderwall sub balke, Noreen, Aschw. Gr. S. 311). Auch in diesem Fall lässt sich die Entlehnungszeit nicht endgültig festsetzen. Prinzipiell könnte nämlich ebenso gut eine alte germanische Entlehnung vorliegen (vgl. z.B. fi. kaura < germ. *hałran-) wie eine jüngere, deren Ausgangspunkt dann der im Altschwedischen regelmässige, auf - $a$ auslautende Synkretismus mehrerer Kasus gewesen wäre: Akk., Gen., Dat. Sg., Akk., Gen. Pl. *balka.

Terho ItKonen 